\title{
Correction to: Not Out of Africa
}

\section{Correction to:}

Chapter 10 in: D. Huylebrouck, Africa and Mathematics, Mathematics, Culture, and the Arts, https://doi.org/10.1007/978-3-030-04037-6_10

The original version of the book was inadvertently published with incorrect figure caption for Fig. 10.4 as "Cartoon about African roots of Egyptian pharaohs". The caption has now been corrected as "Cartoon about African roots of the Egyptian pharaohs based on a drawing in "The Skeptical Inquirer"”.

The updated online version of this chapter can be found at https://doi.org/10.1007/978-3-030-04037-6_10 